\title{
Study on the Evaluation of Mine Environmental Sustainable Development Based on Fuzzy Petri Nets
}

\author{
Chao Zheng, a, *, Changbo Pan ${ }^{2}$, Lan Yu', Jufeng Zhang ${ }^{1,3, b}$, Fengfeng Yang ${ }^{1}$, Rili Yang ${ }^{1}$ \\ ${ }^{1}$ School of Energe Engineering, Longdong University, Qingyang 745000, china \\ ${ }^{2}$ Gansu Energy Qingyang Coal and Electricity Corporation Ltd., Qingyang 745000, china \\ ${ }^{3}$ School of Resource, Environment and Safety Engineering, Hunan University of Science and \\ Technology, Xiangtan 411201, China \\ a513242367@qq.com, ${ }^{b}$ 422622510@qq.com
}

Keywords: Mine environment, Fuzzy Petri Net, Sustainable Development, Evaluation model

Abstract: To evaluate the sustainable development of mine environment, fuzzy Petri nets and variable weight theory were introduced into the study of sustainable development of mine ecological environment. Based on the self-purification and repair functions of the environmental system, a fault tree model of mine environmental pollution was constructed, and the fault tree model was transformed into a mine pollution model based on fuzzy Petri nets. Finally, an inference algorithm and evaluation method based on the fuzzy Petri net model were described. It provides a theoretical basis for the sustainable development evaluation of the mine environment.

\section{Introduction}

The scale mining of mineral resources provides the basic guarantee for the development of the national economy, but the environmental damage and pollution caused cannot be neglected. The development of "green mines" has become the basic goal of today's mine construction, and its core is to achieve the sustainable development of resources and the environment. Due to the large number of elements of the mine environment system, the wide range of impact, the complex relationship, and the difficulty in obtaining evaluation data, the analysis of the reliability and safety of such systems has become increasingly difficult[1-3].Therefore, it is extremely necessary to establish a scientific and reliable mine environmental quality evaluation system, accurately measure the level of sustainable development of the mine, and be able to analyze, analyze and propose measures[4-5].

\section{Fault Tree Analysis of Mine Environment Risk Fuzzy}

\subsection{Purification and repair of contaminants in the atmosphere}

Purification links $A_{11} \cap A_{12}$ indicate that the pollution load control of the mining area meets the secondary standards of the "Ambient Air Quality Standard”. The purification link $\left(\mathrm{A}_{21} \cap \mathrm{A}_{23}\right)$ 
$\left(\mathrm{A}_{22} \cap \mathrm{A}_{23}\right)$ indicates that the pollutants in the mining area are effectively diluted and diffused in the atmosphere. $A_{21} \cap A_{23}$ indicates that the prevailing purging conditions meet certain requirements, and $\mathrm{A}_{22} \cap \mathrm{A}_{23}$ indicate that the prevailing purifying conditions meet certain requirements. The repair links $A_{31}$ and $A_{32}$ indicate that the pollutants in the air of the mining area are absorbed and enriched by functional vegetation $\left(A_{31}\right)$ or are converted into non-toxic or low-toxic substances $\left(A_{32}\right)$ by optical action. The conditions meet the requirements.

\subsection{Purification and repair of pollutants in soil of coal mining area}

Decontamination process $\mathrm{B}_{11} \cap \mathrm{B}_{12}$ indicates that the control of organic pollutants $\mathrm{B}_{11}$ and inorganic pollutants $\mathrm{B}_{12}$ in the soil of the mining area meets the three-level standard of Soil Environmental Quality Standard. Decontamination $\left(\mathrm{B}_{21} \cap \mathrm{B}_{22}\right) \cup\left(\mathrm{B}_{23} \cap \mathrm{B}_{24}\right) \cup\left(\mathrm{B}_{25} \cap \mathrm{B}_{26}\right)$ indicates that the pollutants in the soil solution are separated, diffused, absorbed, decomposed, and converted. The repair links $B_{31}$ and $B_{32}$ indicate that the destroyed vegetation is in good condition.

\subsection{Purification and repair of pollutants in mine water}

Purification links $\mathrm{C}_{11}$ to $\mathrm{C}_{12}$ indicate that the control of harmful substances in the water complies with relevant environmental quality standards. Among them, $\mathrm{C}_{11}$ and $\mathrm{C}_{12}$ are the surface water and groundwater quality that meet the Class III standards of Surface Water Environmental Quality Standard and Groundwater Environmental Quality Standard. Decontamination $\left(\mathrm{C}_{21} \cap \mathrm{C}_{22}\right) \cup$ $\left(\mathrm{C}_{23} \cap \mathrm{C}_{24}\right) \cup\left(\mathrm{C}_{25} \cap \mathrm{C}_{26}\right)$ indicates that the contaminants in the water environment system have good diffusion, diffusion, decomposition, and absorption and enrichment conditions. State $\mathrm{C}_{31}$ indicates the equilibrium of groundwater in the mining area, that is, the replenishment and excretion of groundwater should be balanced.

\subsection{Fault tree of mine environmental pollution}

According to the low sustainability of the mine environment as the top event of the fault tree, according to the series and parallel relationship between the various cleaning links, the layered layer decomposition, the establishment of the mine pollution of the fault tree shown in Figure 1.

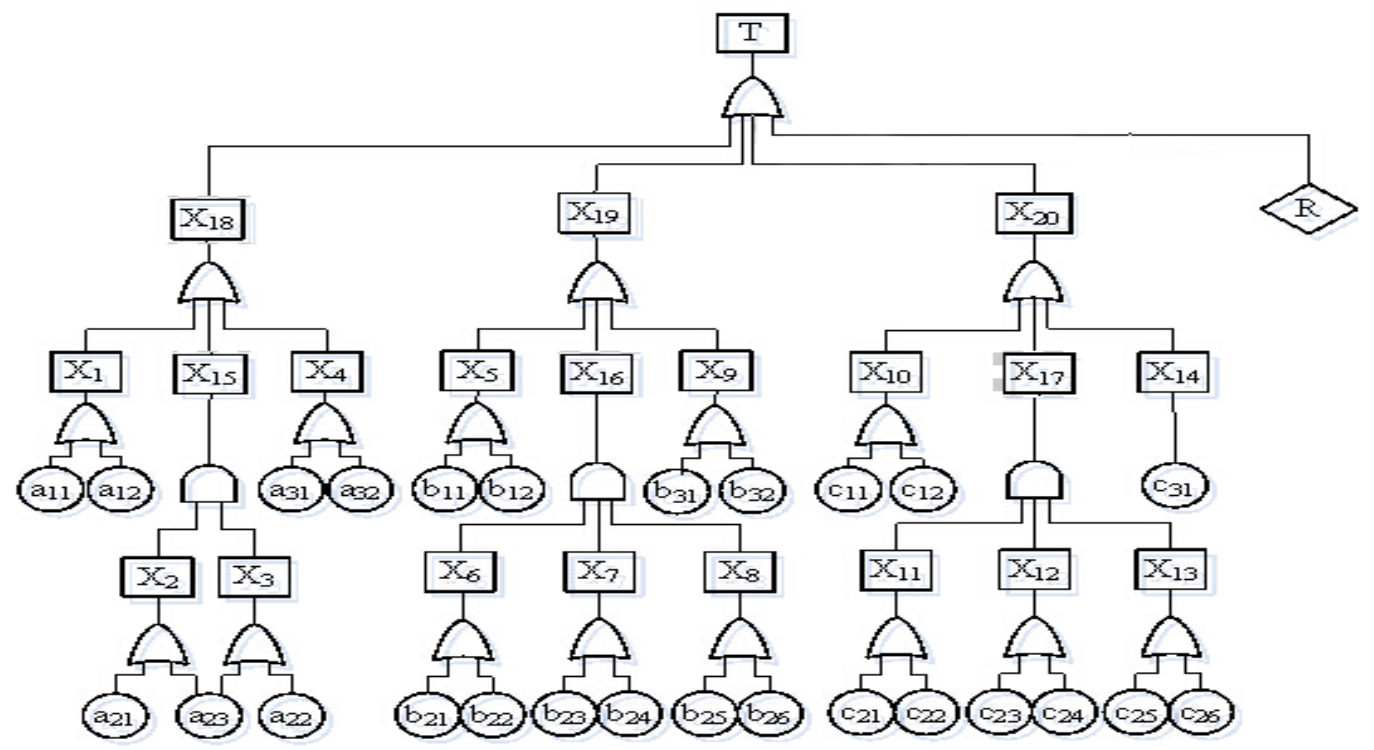

Fig. 1 Mine environment pollution fault tree model 


\section{The Construction of Fuzzy Variable Weighted Petri Net Model}

\subsection{The composition of the model}

The model of mine environmental pollution based on fuzzy Petri nets is nine-tuple: ( $P$, $R, \triangle, \Gamma, C F, \Theta, \gamma, I, \tau)$ :

(1) $P=\left\{p_{1}, p_{2}, \ldots, p_{n}\right\}$ is a finite set of propositional descriptions of mine ecological environmental pollution states (factors). Each proposition corresponds to a library in a Petri net, proposition set $P$ and fault tree model events. Set $A$ one to one correspondence;

(2) $R=\left\{r_{1}, r_{2}, \ldots, r_{m}\right\}$ is a finite set of regularized descriptions of events that result in failure of the system cleanup and repair process. Each rule $r_{i}(i=1,2, \ldots, m)$ corresponds to a Petri net. One of the transitions $t_{i}(i=1,2, \ldots, m)$;

(3) $\Delta=\left\{\delta_{i j}\right\}$, where $i=1,2, \ldots, n, j=1,2, \ldots, m, \delta_{i j} \in[0,1]$ denote the input of the transition $t_{j}$ from the proposition $p_{i}$ to the rule $r_{j}$ intensity, that is, the size of the contribution made by the event $p_{i}$ when the rule $r_{j}$ is established, $n$ ( $n$ is a positive integer) The sum of the weights when the libraries point to the same transition is 1 , which is a known quantity. $\delta_{i j}=\left\{\begin{array}{ll}0 & p_{i} \notin t^{*} t_{j} \\ (0,1] & p_{i} \in t_{j}\end{array} ;\right.$

(4) $\Gamma=\left\{c_{j i}\right\}$, where $j=1,2, \ldots, m, i=1,2, \ldots, n, c_{j i} \in[0,1]$, whose value represents the transition $t_{j}$ corresponding to the rule $r_{j}$ to the library The output intensity of the $p_{i}$, that is, the degree of event $p_{i}$ is promoted after the rule $r_{j}$ is established, and the sum of the output intensities when the same transition points to $n$ ( $n$ is a positive integer) is also a known quantity. among them, $c_{j i}=\left\{\begin{array}{ll}0 & p_{i} \notin t_{j}{ }^{\circ} \\ (0,1] & p_{i} \in t_{j}\end{array}\right.$.

(5) $C F=\operatorname{diag}\left(f_{1}, f_{2}, \ldots, f_{m}\right), f_{j} \in[0,1]$ is the credibility of the rule $r_{j}$, that is, the degree of confidence that the rule $r_{j}$ corresponds to the transition $t_{j}$;

(6) $\Theta=\left(\theta_{1}, \theta_{2}, \ldots, \theta_{n}\right)^{T}, \quad \theta_{i} \in[-1,1], \quad i=1,2, \ldots, n$, is the truth degree of the proposition $p_{i}$, that is, $p_{i}$ corresponds to the corresponding event in the set $A$ Possibility of happening. The value of $\theta_{i}[-1,0)$ indicates that $p_{i}$ has an inhibitory effect on pollution of the mining environment $\left(p_{i} \in U\right)$, and the value $(0,1)$ indicates that $p_{i}$ plays a promoting role $\left(p_{i} \in U^{+}\right)$, and the value $\{0\}$ indicates The role of $p_{i}$ is uncertain $\left(p_{i} \in U^{*}\right)$. The degree of truth of each leaf bank is the probability of occurrence of the basic event in $A$, and $\Theta_{0}$ is the initial trueness vector;

(7) Marker vector for the presence or absence of the proposition $p_{i}(i=1,2, \ldots, n)$ in $P$, $\gamma=\left(\gamma_{1}, \gamma_{2}, \cdots, \gamma_{n}\right)^{T}$. Among them, indicates that $p_{i}$ exists, that is, the represented event may occur; indicates that $p_{i}$ does not exist, that is, the represented event cannot occur. $\gamma_{0}$ is the initial marker vector;

(8) $I: P \times R \rightarrow\{0,1\}$ is an $n \times m$ dimension matrix, indicating that there is a connected arc to the transition from the library. If there is a connection arc from $p_{i}$ to $r_{j}$, then $I\left(p_{i}, r_{j}\right)=1$; otherwise, $I\left(p_{i}\right.$, $\left.r_{j}\right)=0$; where $i=1,2, \ldots, n, j=1,2, \ldots, m$;

(9) $\tau$ is a critical value of the evolution of the inference rule, which represents the minimum probability of occurrence of the event $p_{i}$. Based on the theory of variable weight[7], for any library $p_{i} \in S^{+}$or $p_{i} \in S^{-}$, if $0<\theta_{i}<\tau$ or $-\tau<\theta_{i}<0$, the event $p_{i}$ does not occur, then the proposition is evolved and the degree of truth is $\left|\theta_{i}\right|<\tau$ The library $p_{i}$ evolves to its no-proposition bank, and the link weight $\delta_{i j}$ from transition to transition should also be modified accordingly.

\subsection{The construction of fuzzy Petri net evaluation model}

The construction of the mine environment pollution fuzzy Petri net model is to transform mine environmental pollution fault tree into fuzzy Petri net representation and construct reasoning and 
solving rules. As can be seen from FIG. 1, the fault tree model has only AND (or gate) and OR (or gate) relationships, and the fuzzy Petri net transformation of the AND gate is shown in Figure. 2.

Based on the above-mentioned conversion rules and the definition of the model, the model of Figure 1 is converted to the mine environment pollution fuzzy Petri net model shown in Figure 3.
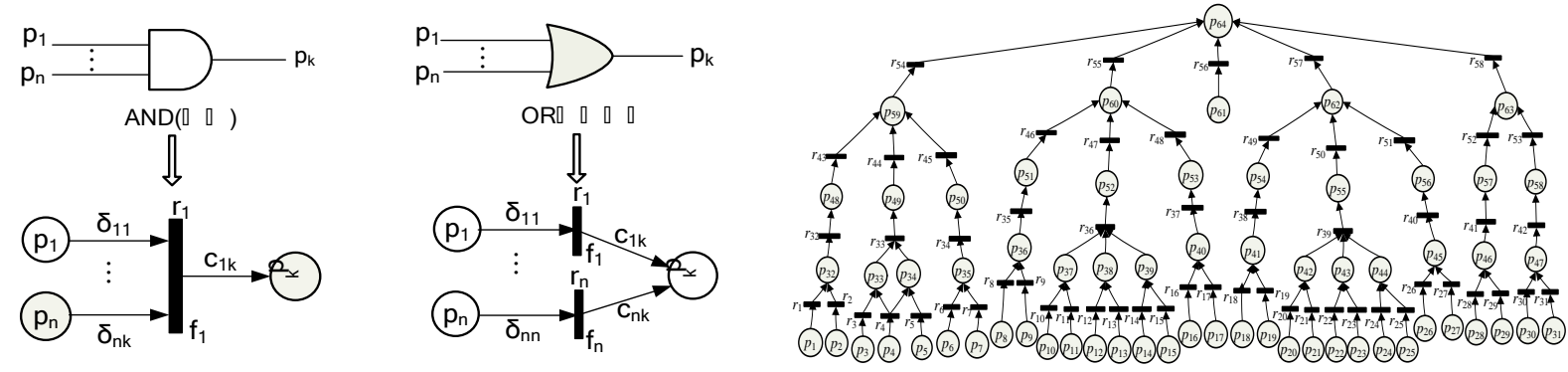

Fig. 2 Transformation of Fuzzy Petri net

Fig. 3 Fuzzy Petri net

\section{Model Inference Algorithm and Evaluation Process}

\subsection{How to calculate the degree of reality of events under two rules}

In any fuzzy Petri net, $t_{j}$ is the transition corresponding to a certain rule, $P$ is the previous set library of $t_{j}$ [8], where the input strength of $p_{i} \in P$ to $t_{j}$ is $\delta_{i j}$, (i=1,2,.., n, then the equivalent fuzzy input confidence $E_{j}$ of $t_{j}$ is:

$$
E_{j}=\sum_{i=1}^{n} \theta_{i} \delta_{i j}
$$

(1) Rule 1. IF $p_{i 1}$ and $p_{i 2}$ and $p_{i 3}$ and $\ldots$ and $p_{i n}$ THEN $p_{k}$, after the transition $\operatorname{ri}(i=1,2, . ., \mathrm{n})$ is stimulated, the truth degree $\theta_{k}$ of the result proposition $p_{k}$ is:

$$
\theta_{k}=\left(\sum_{i=1}^{n} \theta_{i} \delta_{i j}\right) f_{j} \gamma=E_{j} f_{j} \gamma
$$

(2) Rule 2. IF $p_{i 1}$ or $p_{i 2}$ or $p_{i 3}$ or $\ldots$ or $p_{i n}$ THEN $p_{k}$, after the transition $r_{i}(i=1,2, . ., n)$ is stimulated, the truth degree $\theta_{k}$ of the result proposition $p_{k}$ is:

$$
\theta_{k}=\operatorname{Max}\left(\theta_{1} \delta_{1} f_{1} \gamma_{1 k}, \theta_{2} \delta_{2} f_{2} \gamma_{2 k}, \ldots, \theta_{n} \delta_{n} f_{n} \gamma_{n k}\right)=\operatorname{Max}\left(E_{1} f_{1} \gamma_{1 k}, E_{2} f_{2} \gamma_{2 k}, \ldots, E_{n} f_{n} \gamma_{n k}\right)
$$

\subsection{Construction model reasoning algorithm}

In the model, $P$ and $R$ are state proposition sets and behavior rule sets, respectively, where $|P|=n,|R|=m$. Let the equivalent fuzzy input confidence of each transition in the model be $E=\left(e_{1}, e_{2}, \ldots, e_{m}\right)^{\mathrm{T}}$. The reasoning algorithm is as follows:

Step1 Initialize $\Theta, C F, \Delta, \Gamma, I, \gamma, \tau$, set $k=1$;

Step2 judge according to the trigger rule of the transition, if it is the case 1, go to Step3; if it is the case 2, then execute Step4;

Step3 Calculate the degree of authenticity of the output library after the transition excitation based on rules 1 and 2 and update the truth degree set $\Theta$; go to Step 8;

Step4: Perform rule evolution and change of weights, calculate the trueness $\theta_{i}^{\prime}$ and weight of the $p_{i}$ after variable weight processing $\delta_{i k}^{\prime}$, and obtain a new trueness set and input intensity matrix $\Theta_{k}^{\prime}$ 、 $\Delta^{\prime}[6] ;$

Step5 Calculate the evolutionary equivalent fuzzy input credibility from equation (1) and obtain a new proposition trueness set [9]. 


$$
\Theta_{k}^{\prime \prime}=\Gamma^{\mathrm{T}} \otimes\left(E_{k}{ }^{\prime \mathrm{T}} C F\right)^{\mathrm{T}}
$$

Step6 Calculate the new round of authenticity to ensure that the absolute value of the trueness of the proposition corresponding to the library is not reduced;

$$
\Theta_{k}=\Theta_{k}^{\prime \prime} \oplus \Theta_{k}^{\prime}
$$

Step7 Update flag vector $\gamma_{k}$ : If the transition $r_{j}$ triggers, the flag value of the succeeding library closest to the transition $r_{j}$ is set to 1 , otherwise it is set to 0 ;

Step8 when $\Theta_{k}=\Theta_{k-1}$, reasoning is over, and assignment $\Theta=\Theta_{k}$; otherwise, go to Step9;

Step9 set $k=k+1$, go to Step2[10].

\subsection{Evaluation of sustainable development of mine ecological environment}

Based on the propositional truth degree $\Theta$ obtained by the above inference algorithm, each trueness value $\Theta$ is divided into two cases: $\tau \leq \theta_{i} \leq 1$ or $-1 \leq \theta_{i} \leq-\tau$. In order to facilitate the assessment of the degree of sustainable development, the formula (6) will be converted to the corresponding sustainable development value $v_{i}$ of each subsystem, The value of $v_{i}$ represents the sustainable development and development level, and $v_{i} \geq 0.6$ is the intermediate sustainable development level. Stronger sustainability. Based on this, we will make an assessment of the sustainable development level of each subsystem and the entire mine ecological environment, and make a reason analysis and provide feedback.

$$
v_{i}=\left\{\begin{array}{lr}
1-\theta_{i} & \theta_{i} \geq \tau \\
\left|\theta_{i}\right| & \theta_{i} \leq-\tau
\end{array}\right.
$$

\section{Conclusion}

Based on the self-purification and restoration functions of the environmental system, this paper establishes a fault tree model of mine wastewater, harmful gases and solid waste, and introduces the fuzzy Petri net and variable weight theory into the study of the sustainable development of mine ecological environment. At the same time, the fault tree model is transformed into the mine pollution model of the fuzzy Petri net. Finally, the inference algorithm and evaluation method based on the fuzzy Petri net model are described. The sustainable development and continuous level of the mine's ecological environment are evaluated through the mine ecological environment sustainability value.

\section{Acknowledgments}

This work was financially supported by 2018 Gansu Provincial Safety Science and Technology Project [GAJ00017] and Longdong University Youth Science \& Technology Innovation Project [XYZK1611] fund.

\section{References}

[1] Wei Yufeng, Kan Shulin, Ren Yizhou. Petri nets-based fault tree analysis method for complex manufacturing systems [J]. Machinery Design \& Manufacture, 2010, (7):34-39.

[2] V.E. Johnson, A. Moosman, P. Cotter.A Hierarchical Model for Estimating the Early Reliability of Complex Systems. Transaction on Reliability,vol,2005,54(2):224-228.

[3] S.K. Chen, T.K. Ho, B.H. Mao. Reliability evaluations of railway power supplies by fault-tree analysis. IET Electr. Power, Appl,2007,1(2):161-172. 
[4] B. Gollomp. Quality and Reliability Facilitator-FMEA. Instrumentation\& Measurement Magazine. Volume 11, Issue 2, April,2008 Page(S):58-59.

[5] Huang Guangqiu, Zhu Huaping, Zheng Yanquan. Study on Fuzzy A nalysis of Accidents Based on Fuzzy Petri Net from Fault Tree[J]. Journal of Hunan University of Science \& Technolog. 2006,21(2):34-39.

[6] Jang Zhibin. Petri-net and Its Application in Manufacture System Modeling and Control [M]. Beijing: China Machine Press 2004.

[7] Lin Lizhong, Zhu Bin. Study on a New Model of Regional Environmental Air Quality Risk Assessment Based on Reliability Theory[J]. China Population, Resources and Environment, 2006,16(1):62-65.

[8] Huang Guangqiu, Wang Jincheng. Consistent varying-weight fuzzy Petri net attack model based on both-branch fuzzy set [J]. Journal of Computer Applications,2009,29(2):529-534.

[9] Lu Qiuqin, Liu Haozheng, Duan Wenqiang. Study on Tenderee Trust Risk Evaluation Model Based on Fuzzy Petri Net[J]. Zhengzhou Univercity, (Nat, Sci, Ed),2014, (1):120-125.

[10] Wang Yang, Lin Chuang, Qu Yang, Li Yajuan. Consistent Fuzzy Petri Nets Model for Logic Programs with Negation[J]. ACTA ELECTRONICA SINICA, 2006,34(11):1955-1960. 